\title{
Tests for Price Indices in a Dynamic Item Universe
}

\author{
Li-Chun Zhang ${ }^{1}$, Ingvild Johansen ${ }^{2}$, and Ragnhild Nygaard ${ }^{2}$
}

\begin{abstract}
There is generally a need to deal with quality change and new goods in the consumer price index due to the underlying dynamic item universe. Traditionally axiomatic tests are defined for a fixed universe. We propose five tests explicitly formulated for a dynamic item universe, and motivate them both from the perspectives of a cost-of-goods index and a cost-of-living index. None of the indices that are currently available for making use of scanner data satisfies all the tests at the same time. The set of tests provides a rigorous diagnostic for whether an index is completely appropriate in a dynamic item universe, as well as pointing towards the directions of possible remedies. We thus outline a large index family that potentially can satisfy all the tests.
\end{abstract}

Key words: Axiomatic test; cost of goods; cost of living; quality change; new goods.

\section{Introduction}

The failure to take full account of quality change and new goods is one of the important sources of the potential bias of the consumer price index (CPI). See for example CPI Manual. By convention the term 'quality change' pertains to situations where new products, models, services and so on are compared to the old items they are deemed to replace, whereas new goods are thought of as wholly new types of items, such as when microwave ovens were first introduced in the market. The cause of the problems is thus the same, namely, the item universe of the CPI is dynamic such that one needs to compare the prices of different sets of items over time.

In recent years, greater uses are made of scanner data, where one has access to the average transaction price and quantity of each item (identified by the Global Trade Item Number, GTIN) over the period of data collection. A key research question is how to make use of the quantity data that have become available below the level of elementary aggregation. Explicit replacement of the old items by the new ones for matched price comparisons becomes exceedingly resource demanding, if applied to all the available items. The hedonic methods are often infeasible due to the lack of characteristics data. The research is active at the moment regarding index formulae that are only based on the price and quantity data. See for example Chessa et al. (2017), Dalén (2017), Diewert and Fox (2017), Zhang et al. (2017). However, there is currently a lack of consensus on how to evaluate them. While the choice of index formula may not seem to have a big impact on certain consumption segments, including food (e.g., Chessa et al. 2017; Zhang et al. 2017),

1 University of Southampton, Highfield, Southampton, SO17 1BJ, United Kingdom. Email: L.Zhang@ soton.ac.uk

2 Statistics Norway, P.O. Box 2633 St. Hanshaugen NO-0131 Oslo, Norway. Emails: ingvild.johansen@ssb.no and ragnhild.nygaard@ssb.no 
the choice does matter in many other situations, such as when there is a high item churn rate, and/or where the prices of goods undergo strong decline during their respective life spans. See for example Chessa et al. (2017) for some relevant empirical evidence on men's t-shirts and television.

The test approach provides a valuable theoretical framework for index numbers, in addition to the economic and stochastic approaches. However, the traditional axiomatic tests (Fisher 1922; CPI Manual 2004, chap. 16) are all defined for a fixed item universe. Tests for multilateral index have been considered for international comparisons; see, for example Diewert (1999) and Balk (1996, 2001). These tests have been adapted in terms of price and quantity comparisons over time (ABS 2016), though without addressing the issue that the universe is actually dynamic over time. None of the major multilateral methods considered in ABS (2016) passes all the tests.

In this article, we propose five tests explicitly formulated for a dynamic item universe. The tests will be motivated both from the perspectives of a cost-of-goods index (COGI) and a cost-of-living index (COLI). As mentioned in paragraph 16.2 of the CPI manual, "different price statisticians may have different ideas about which tests are important [...]" Moreover, there is generally the possibility that a test (or axiom), which otherwise seems attractive, may be in conflict with the principles of another theoretical approach to index numbers. Therefore, the plausibility of a test is clearly strengthened if it can be motivated from both the COGI and COLI perspectives.

Notice that definition of a COGI in a dynamic item universe is not evident. According to Schultze and Mackie (2002), COGI "measures the change in expenditures required by a household to purchase a fixed-weight basket of goods and services when prices change between some initial reference period and a subsequent comparison period", whereas COLI "measures the change in expenditures a household would have to make in order to maintain a given standard of living". Accordingly, we shall maintain that a COGI is calculated based on a fixed-weight basket of items despite the fact that the item universe is dynamic over time, regardless of how one settles the choice of the items to be included in such a fixed basket.

The proposed tests will be applied to the indices for scanner data, which have so far received the most attention. These include the Geary-Khamis (GK) index (Geary 1958; Chessa 2016), the generalised unit value (GUV) index family (Dalén 2001; De Haan 2001; Auer 2014), the weighted geometric means (WGM) index family including the time product dummy (TPD) index (De Haan and Krsinich 2014), and the Gini-Eltetö-KovesSzulc (GEKS) index (Ivancic et al. 2011). It is shown that none of them satisfy all five tests at the same time. We will outline a large index family, which includes the GUV index family as a subclass in general and the Fisher index as a special case of bilateral index in a fixed item universe. As will be explained, this family of indices can potentially satisfy all the tests, thus providing impetus for future research.

Overall, extending the test approach to a dynamic universe has at least three advantages. (i) Formulating explicit tests strengthens the rigour of investigation, because a test result is dichotomous by construction whereas an empirical comparison may be inclusive on its own. (ii) It can help to clarify certain conceptual ambiguities. For instance, transitivity can obviously prevent chain drifting in a fixed universe. However, as we shall discuss in Subsection 2.3, a rigorous definition of transitivity is impossible in 
a dynamic universe. The proposed tests can help to articulate the intuition against chain drifting and to avoid an index method that suffers from chain drifting. (iii) Where an index fails a test, it points to the direction in which the index can possibly be improved. For instance, it will be shown that the bilateral GK index fails the responsiveness test T5 in general.

The rest of the article is organised as follows. In Section 2, the necessary notations and concepts are introduced, following which the tests are defined and explained, including a discussion of why some other seemingly obvious tests are not included in the proposal. In Section 3 the tests are applied to the indices mentioned above. Finally, an index family is outlined in Section 4 which potentially can satisfy all the tests, and a few concluding remarks are given in Section 5.

\section{Tests for a Dynamic Item Universe}

\subsection{Notations}

Let us formally introduce the notations and terms that are necessary for a dynamic item universe. Consider a given item universe in period $t$, denoted by $U_{t}=\left\{1,2, \ldots, N_{t}\right\}$, which constitutes a subset (or sector) of the entire CPI item universe. For instance, $U_{t}$ may refer to all food and non-alcoholic beverages sold at supermarkets, or all personal computers sold at electro warehouses, and so on. Notice that generally we allow the items to be specific for an outlet or chain. For example, the same mobile phone model sold at two different outlets can be treated as two different items. Assume that one has available the unit value price $p_{i}^{t}$ and the transaction total $v_{i}^{t}$, for each $i \in U_{t}$, where $v_{i}^{t}=p_{i}^{t} q_{i}^{t}$ and $q_{i}^{t}$ denotes the transaction quantity of the item. Note that the unit value price refers to the average transaction price of an item in the period $t$, where the actual transaction price may change over the period. Denote the set of quantities by $q\left(U_{t}\right)=$ $\left\{q_{i}^{t} ; i \in U_{t}\right\}$ and the set of prices by $p\left(U_{t}\right)=\left\{p_{i}^{t} ; i \in U_{t}\right\}$. Denote all the data from period $t$ by

$$
D_{t}=D\left(U_{t}\right)=\left\{p\left(U_{t}\right), q\left(U_{t}\right) ; U_{t}\right\}
$$

Denote by $\left(U_{0}, U_{t}\right)$ the comparison universe, for which we seek a price index from 0 to $t$, denoted by $P^{0, t}$. We refer to 0 as the base period and $t$ the statistical (or current) period. Denote by $U_{R(0, t)}=\left\{U_{r} ; r \in R\right\}$ the reference universe, where $R$ is the set of reference periods involved, and the notation $U_{R(0, t)}$ emphasises the dependence of $R$ on the comparison universe - we may suppress $(0, t)$ whenever such an emphasis is unnecessary. We consider price indices that are functions of the data $D_{R}=D\left(U_{R}\right)=\left\{D_{t} ; t \in R\right\}$, which can be given as

$$
P^{0, t}=f\left(D_{R}\right) .
$$

Two choices of the reference universe $U_{R}$ are most immediate, that is

$$
\begin{aligned}
& R_{B}=\{0, t\} \quad \text { and } \quad U_{R_{B}}=U_{0} \cup U_{t}, \\
& R_{M}=\{0,1, \ldots, t\} \quad \text { and } \quad U_{R_{M}}=U_{0} \cup U_{1} \cup \cdots \cup U_{t},
\end{aligned}
$$


where $R_{B}$ implies direct comparison between 0 and $t$, for example December 2014 (0) and July $2015(t)$, and $R_{M}$ implies all the periods from 0 to $t$, for example December 2014 to July 2015. An index based on the reference universe $U_{R_{B}}$ is referred to as a bilateral index; it is multilateral otherwise.

We notice that, when 0 is fixed as $t$ moves forward, the choice of $R_{M}$ above corresponds to using a fixed base expanding window. It is, of course, possible to use another reference set, such as a rolling window of fixed length counting backwards from $t$, which includes period 0 . Moreover, the definition of reference universe formally covers the use of chained index, for example when $t$ moves beyond a year-length window into the next one. We do not discuss chained index explicitly in the sequels, because a chained index would fail a test if any part of the chain fails that test. Finally, unless otherwise noted, one may keep in mind the reference universe $R_{M}$ above when reading this article, because the conclusions then also hold for other choices of $R_{M}$.

\subsection{The Tests}

In this subsection we formulate five tests for a dynamic item universe, and motivate them from both the COGI and COLI perspectives. Note that in the case of fixed item universe and $R=R_{B}$, both the tests $\mathrm{T} 1$ and $\mathrm{T} 2$ reduce to well-known tests for a fixed item universe. However, more generally, $U_{0}=U_{t}$ represents the special case where a dynamic universe may sometimes return to the same state of affairs, even when $U_{r} \neq U_{0}=U_{t}$ for all $r \neq 0, t$. By the tests $\mathrm{T} 1$ and $\mathrm{T} 2$ we require that a dynamic-universe index should not then have counter-intuitive properties.

Let $U_{0 t}=U_{0} \cap U_{t}$ be the persistent item universe at 0 and $t$, and $U_{t \backslash 0}=U_{t} \backslash U_{0}$ the set of birth items and $U_{0 \backslash t}=U_{0} \backslash U_{t}$ that of the death items. The item universe is (always) fixed if $U_{t}=U_{0}=U_{0 t}$ for any $t \neq 0$, in which case $U_{t \backslash 0}=U_{0 \backslash t}=\emptyset$; it is dynamic otherwise.

Identity test (T1) If $U_{0}=U_{t}$ and $p_{i}^{0} \equiv p_{i}^{t}$ for all $i \in U_{0}$, then $P^{0, t}=1$.

Since the item universe is the same at 0 and $t$, so must be the items eligible for a COGI, when the comparison universe is $\left(U_{0}, U_{t}\right)$. Thus, despite the changes of item universe that take place between the two time points, where $t>1$, the identity test can be motivated for a COGI. Now that all the prices are the same at 0 and $t$, a COGI index must necessarily be 1 , regardless of how the reference quantities of the goods are calculated. Therefore, a cost-of-goods index should satisfy the identity test. Next, consider a COLI. Let $V^{0, t}=$ $\sum_{i \in U_{t}} q_{i}^{t} p_{i}^{t} / \sum_{i \in U_{0}} q_{i}^{0} p_{i}^{0}$ be the ratio of total expenditures. Under the stipulated setting, it is obviously possible to maintain the same utility without changing the total expenditure. Thus, insofar as $V^{0, t} \neq 1$, all the change in expenditure must be attributed to the change in utility but not prices, under the assumption of rational consumer behaviour. A COLI should therefore be equal to 1 .

Despite the item universe varies at other points than 0 and $t$, an index that satisfies the identity test is said not to drift in this situation, which can be used to examine for example, a multilateral index. We notice that chain drift is often contrasted with transitivity. However, as will be discussed in Subsection 2.3, we find it difficult to define a transitivity 
test for a dynamic item universe. For the moment the identity test $\mathrm{T} 1$ is the only test we have with respect to chain drift.

Fixed basket test (T2) If $U_{0}=U_{t}$ and $q_{i}^{0} \equiv q_{i}^{t}$ for all $i \in U_{0}$, then $P^{0, t}=V^{0, t}$.

The test is obvious for a COGI. It can easily be satisfied by any bilateral COGI. Otherwise, in a dynamic universe, one may have $q_{i}^{r} \neq q_{i}^{0}$, for $0<r<t$, so that the item reference quantity can differ from $q_{i}^{0}=q_{i}^{t}$, and a fixed basket-of-goods index may not be equal to $V^{0, t}$. It follows that, in order for a COGI to satisfy $\mathrm{T} 2$, one may need to avoid the use of multilateral indices. The test $\mathrm{T} 2$ is readily motivated for a COLI. Given the quantities $q\left(U_{t}\right)$ are actually the same as $q\left(U_{0}\right)$, no utility adjustment of $V^{0, t}$ is needed, and a COLI at the observed utility is equal to $V^{0, t}$.

Upper bound test (T3) If $U_{0} \subseteq U_{t}$, and $p_{i}^{t} \leq p_{i}^{0}$ for all $i \in U_{0}$, then $P^{0, t} \leq 1$.

That is, the item universe may be constant if $U_{0}=U_{t}$ or strictly expanding if $U_{0} \subset U_{t}$, and the price of each persistent item is the same or reduced, that is $p_{i}^{t} \leq p_{i}^{0}$ for all $i \in U_{0 t}=U_{0}$. The proof is rooted in the economic theory of Preferences. Basically, the premises of test T3 set up a situation which guarantees that a consumer can maintain their utility without increasing the total expenditure. Given this is the case, the intuition is that any rational change of consumption can only occur because the consumer has found a better utility-cost ratio, as the choices increase with the expanding item universe. The arguments are given below in details.

Firstly, suppose substitution does not occur, in which case $q_{i}^{t}=q_{i}^{0}$ for all $i \in U_{0}$ and $q_{i}^{t}=0$ for all $i \in U_{t \backslash 0}$, even if $U_{t \backslash 0}$ is nonempty. The actual comparison universe reduces then to $U_{0 t}$, so that the test $\mathrm{T} 2$ applies, yielding $P^{0, t}=V^{0, t} \leq 1$ under the stipulated setting. Next, suppose substitution occurs only among the persistent items, i.e., $q_{i}^{t}=0$ for $i \in U_{t \backslash 0}$ and $q_{i}^{t} \neq q_{i}^{0}$ for some $i \in U_{0 t}$. Substitution can be accounted for from the perspective of COLI. Given the actual $\left\{q_{i}^{t} ; i \in U_{0 t}\right\}$ and the corresponding utility at $t$, it cannot cost less for the same $\left\{q_{i}^{t} ; i \in U_{0 t}\right\}$ at 0 since $p_{i}^{t} \leq p_{i}^{0}$ for all $i \in U_{0 t}$. It follows that a COLI must be less than or equal to 1 . Finally, suppose substitution also involves the items in $U_{t \backslash 0}$. Let $\left\{\tilde{q}_{i}^{t} ; i \in U_{0 t}\right\}$ be a hypothetical set of persistent items that would have yielded the same utility as the actual $\left\{q_{i}^{t} ; i \in U_{t}\right\}$. Owing to rational behaviour, the expenditure of $\left\{\tilde{q}_{i}^{t} ; i \in U_{0 t}\right\}$ at $t$ cannot be less than the actual expenditure of $\left\{q_{i}^{t} ; i \in U_{t}\right\}$; whereas the expenditure of $\left\{\tilde{q}_{i}^{t} ; i \in U_{0 t}\right\}$ at 0 cannot be less than that at $t$. It follows again that a COLI must be less than or equal to 1 .

Lower bound test (T4) If $U_{t} \subseteq U_{0}$, and $p_{i}^{t} \geq p_{i}^{0}$ for all $i \in U_{t}$, then $P^{0, t} \geq 1$.

That is, the item universe may be constant or strictly shrinking, and the price of each persistent item is the same or increased. Firstly, suppose substitution does not occur, in which case $q_{i}^{t}=q_{i}^{0}$ for all $i \in U_{0 t}$ and $q_{i}^{t}=0$ for all $i \in U_{0 \backslash t}$. Then, the comparison universe reduces to $U_{0 t}$, and the test T2 applies, yielding $P^{0, t}=V^{0, t} \geq 1$ under the stipulated setting. Next, suppose substitution occurs only among the persistent items. Given any actual $\left\{q_{i}^{t} ; i \in U_{0 t}\right\}$ and the corresponding utility at $t$, it cannot cost more for the same $\left\{q_{i}^{t} ; i \in U_{0 t}\right\}$ at 0 since $p_{i}^{0} \leq p_{i}^{t}$ for all $i \in U_{0 t}$. It follows that a COLI must be greater than or equal to 1 . Finally, suppose substitution also involves the items in $U_{0 \backslash t}$. Let $\left\{\tilde{q}_{i}^{0} ; i \in U_{0 t}\right\}$ be a hypothetical set of persistent units that would have yielded the same 
utility as the actual $\left\{q_{i}^{0} ; i \in U_{0}\right\}$. The expenditure of $\left\{\tilde{q}_{i}^{0} ; i \in U_{0 t}\right\}$ at 0 cannot be less than the actual expenditure of $\left\{q_{i}^{0} ; i \in U_{0}\right\}$; whereas the expenditure of $\left\{\tilde{q}_{i}^{0} ; i \in U_{0 t}\right\}$ at $t$ cannot be less than that at 0 . It follows again that a COLI must be greater than or equal to 1 .

Under the setting of test T3, there exists a clear downwards trend of the prices of persistent items. We should have $P^{0, t} \leq 1$ even if this leads to an increase of expenditure, that is $V^{0, t}>1$. Under the setting of test $\mathrm{T} 4$, there exists a clear upwards trend of the prices of persistent items. We should have $P^{0, t} \geq 1$ even if the price increase causes the expenditure to drop, that is $V^{0, t}<1$.

It is possible to formulate two somewhat sharper versions of the tests $\mathrm{T} 3$ and $\mathrm{T} 4$, respectively, according to which $P^{0, t}$ can possibly deviate from 1 in a particular direction depending on whether the item universe is expanding or shrinking, when all the prices of persistent items remain the same. These are thus clearly the implications of the fact that the item universe is dynamic.

Test t3 If $U_{0} \subset U_{t}$ and $p_{i}^{0}=p_{i}^{t}$ for all $i \in U_{0}$, then $P^{0, t} \leq 1$.

Test $t 4$ If $U_{t} \subset U_{0}$ and $p_{i}^{0}=p_{i}^{t}$ for all $i \in U_{0}$, then $P^{0, t} \geq 1$.

Responsiveness test (T5) For $U_{0} \neq U_{t}, P^{0, t}$ should not always reduce to $f\left(D_{0 t}\right)$, where $D_{0 t}=D\left(U_{0 t}\right)$.

That is, one should not always be able to reduce a COGI to a fixed-basket index, where the basket items only consist of the persistent items. This is necessary for any COGI that, in principle, can be applied to a dynamic item universe; whereas one should not always be able to reduce a COLI to $f\left(D_{0 t}\right)$, since it must allow for substitution that involves the birth and death items.

One can formulate a sharper version of the test T5, where $p_{i}^{0}=p_{i}^{t}$ for all $i \in U_{0 t}$. According to T1, we have then $P^{0, t}\left(D_{0 t}\right)=1$, which is the price index of the persistent item universe $U_{0 t}$. Any $P^{0, t}$ that is always equal to 1 , regardless of $D\left(U_{t \backslash 0}\right)$ or $D\left(U_{0 \backslash t}\right)$, is not responsive.

Test t5 For $U_{0} \neq U_{t}$, if $p_{i}^{0}=p_{i}^{t}$ for all $i \in U_{0 t}$, then $P^{0, t}$ cannot always be equal to 1 , regardless of $D\left(U_{t \backslash 0}\right)$ and $D\left(U_{0 \backslash t}\right)$.

\subsection{Discussion}

The proposed tests are certainly not the only ones possible. However, we have not included any other tests here for several reasons. Firstly, it would have made little difference to include a test that is easily satisfied, an example of which is the time reversal test. Next, some tests seem no longer relevant given the birth and death items. The quantity reversal and price reversal tests are two such examples. Moreover, we have excluded some familiar tests and only retained a sharper version of them. An example is the proportionality test. Since the proportionality test implies the identity test $\mathrm{T} 1$, the latter is sharper than the former, in the same sense that the test $\mathrm{t} 3$ is sharper than T3. Finally, there may be additional concerns that make a test difficult to formulate. An example is the transitivity test, as we discuss below.

Conceptually, an index is transitive if $P^{0, t}=P^{0, r} P^{r, t}$ for any $r \neq 0, t$, provided all the three indices in the form of (1) are calculated in the same way, which generally involves 
three different reference universes $U_{R(0, t)}, U_{R(0, r)}$ and $U_{R(r, t)}$ when the item universe is dynamic. Now, a motivation for transitivity is to prevent chain drifting, when chaining is used to alleviate the difficulty one would encounter in making direct price comparisons between $U_{0}$ and $U_{t}$, where $U_{t \backslash 0}$ and $U_{0 \backslash t}$ may be non-negligible in size compared to $U_{0 t}$. However, in order to verify whether or not chain drifting is the case, one must compare the chained index to the direct index that could have been calculated between 0 and $t$. Thus, one cannot avoid running into the same difficulty that has motivated the chaining in the first place. To push the difficulty to the logical extreme, suppose $U_{0} \cap U_{t}=\emptyset$, i.e., the item universe is completely renewed. What are the conditions of non-drifting, or transitivity, in this case?

From a more pragmatic point of view, the GEKS index has been adapted for temporal price comparisons (Ivancic et al. 2011), as a means to achieve transitivity, provided $P^{0, r}$ are well-defined and time reversible for any $0<r \leq t$. However, international comparisons have a fixed reference set of countries (or regions), and are symmetric in the sense that any two countries are eligible for comparison. The temporal extension has a direction and is ever-changing. Regarding the direction of time, it seems counter-intuitive to require $P^{0, r} P^{r, t}=P^{0, t}$, for an arbitrarily chosen period $r$, where $r<0$ or $r>t$. Regarding the changing reference set, the GEKS index $P^{0, t}$ calculated at $t$ will generally differ to $P^{0, t}$ calculated at $t^{\prime}$, for $t^{\prime}>t$. It follows from both accounts that in reality the disseminated GEKS index is nevertheless intransitive - see Subsection 3.4 for details.

\section{Application}

The test results are summarised in Table 1 . We show that the constant-value adjustment of the GK index, which is necessary in the context of international comparisons involving different currencies, results in unresponsiveness if $R=R_{B}$. Dropping the constant-value adjustment yields the modified GK (MGK) index, which is a member of the GUV index family. We consider also the WGM index family, which includes the TPD index as a special case in a dynamic universe and the Törnqvist index as a special case in a fixed universe. Finally, we discuss the GEKS index, which can use any bilateral time-reversible index as its component.

Table 1. Summary of test results.

\begin{tabular}{|l|c|c|c|c|l|}
\hline GK & $\begin{array}{c}\text { Identity } \\
\text { Yes if } R_{B} \\
\text { No if } R_{M}\end{array}$ & Yes & Yes & Yes & No if $R_{B}$ \\
\hline $\begin{array}{l}\text { GUV } \\
\text { (MGK) }\end{array}$ & $\begin{array}{c}\text { Yes if } R_{B} \\
\text { No if } R_{M}\end{array}$ & Yes & Yes & Yes & $\begin{array}{l}\text { No if } R_{B} \text { and in } \\
\text { Setting of } \mathrm{t} 3 \\
\text { (or } \mathrm{t} 4)\end{array}$ \\
\hline $\begin{array}{l}\text { WGM } \\
(\mathrm{TPD})\end{array}$ & $\begin{array}{c}\text { Yes if } R_{B} \\
\text { No if } R_{M}\end{array}$ & No & Yes & Yes & $\begin{array}{l}\text { No if } R_{B} \text { and in } \\
\text { Setting of } \mathrm{t} 3 \\
\text { (or } \mathrm{t} 4)\end{array}$ \\
\hline GEKS & No & No & No & No & Yes \\
\hline
\end{tabular}




\subsection{The GK Index}

Deflating a constant-value reference-price quantity index yields the GK index:

$$
\begin{gathered}
P_{G K}^{0, t}=V^{0, t} / Q^{0, t} \quad \text { and } \quad Q^{0, t}=\sum_{i \in U_{t}} p_{i} q_{i}^{t} / \sum_{i \in U_{0}} p_{i} q_{i}^{0}, \\
p_{i}=\sum_{r \in R_{i}} \frac{p_{i}^{r}}{P^{0, r}} q_{i}^{r} / \sum_{r \in R_{i}} q_{i}^{r},
\end{gathered}
$$

where the observed price $p_{i}^{r}$ is adjusted to a constant-value price by $P^{0, r}$, and $R_{i}$ contains the periods at which the item $i$ is in the market.

The GK index given by (2) and (3) does not satisfy the identity test T1 except when $R=R_{B}$, in which case $p_{i}=p_{i}^{0}=p_{i}^{t}$. Next, it satisfies the fixed-basket test T2 since $Q^{0, t}=1$. Thirdly, it satisfies the upper bound tests T3 and 3 , provided $p_{i}^{t} \leq p_{i} \leq p_{i}^{0}$ by (3), such that

$$
Q^{0, t}=\frac{\sum_{j \in U_{0}} p_{j} q_{j}^{t}+\sum_{j \in U_{t \backslash 0}} p_{j}^{t} q_{j}^{t}}{\sum_{j \in U_{0}} p_{j} q_{j}^{0}} \geq \frac{\sum_{j \in U_{0}} p_{j}^{t} q_{j}^{t}+\sum_{j \in U_{t \backslash 0}} p_{j}^{t} q_{j}^{t}}{\sum_{j \in U_{0}} p_{j}^{0} q_{j}^{0}}=V^{0, t}
$$

Moreover, it satisfies the lower bound tests T4 and t4, since we then have $Q^{0, t} \leq V^{0, t}$.

When it comes to the test T5, below we give a proof that the bilateral GK index is generally unresponsive. Previously, Geary (1958) pointed out that for spatial comparison with a matched commodity universe between two countries, the GK index reduces to a fixed weights price index, where the weight of a commodity is given by the harmonic means of its quantities in both countries. As we now demonstrate, the same result holds even when the two universes are not completely matched. In the present notation, the bilateral GK index can be given as $E_{0} / E_{t}$, which together with the reference prices satisfy the following linear system

$$
\begin{aligned}
& p_{i}= \begin{cases}E_{0} \frac{p_{i}^{0} q_{i}^{0}}{q_{i}^{0}+q_{i}^{t}}+E_{t} \frac{p_{i}^{t} q_{i}^{t}}{q_{i}^{0}+q_{i}^{t}} & \text { if } i \in U_{0 t} \\
E_{0} p_{i}^{0} & \text { if } i \in U_{0 \backslash t}=U_{0} \backslash U_{0 t} \\
E_{t} p_{i}^{t} & \text { if } i \in U_{t \backslash 0}=U_{t} \backslash U_{0 t}\end{cases} \\
& E_{r}=\sum_{i \in U_{r}} p_{i} q_{i}^{r} / \sum_{i \in U_{r}} p_{i}^{r} q_{i}^{r} \quad \text { for } r=0, t
\end{aligned}
$$

That the GK index reduces to a matched-universe index can be seen in the following:

$$
\begin{aligned}
\left(\sum_{i \in U_{t}} p_{i}^{t} q_{i}^{t}\right) E_{t} & =E_{0}\left(\sum_{i \in U_{0 t}} \frac{p_{i}^{0} q_{i}^{0}}{q_{i}^{0}+q_{i}^{t}} q_{i}^{t}\right)+E_{t}\left(\sum_{i \in U_{0 t}} \frac{p_{i}^{t} q_{i}^{t}}{q_{i}^{0}+q_{i}^{t}} q_{i}^{t}+\sum_{U_{t} \backslash 0} p_{i}^{t} q_{i}^{t}\right) \\
& \Rightarrow E_{t}\left(\sum_{i \in U_{0 t}} \frac{q_{i}^{0} q_{i}^{t}}{q_{i}^{0}+q_{i}^{t}} p_{i}^{t}\right)=E_{0}\left(\sum_{i \in U_{0 t}} \frac{q_{i}^{0} q_{i}^{t}}{q_{i}^{0}+q_{i}^{t}} p_{i}^{0}\right) .
\end{aligned}
$$




\subsection{The GUV Index Family}

Removing the constant-value adjustment via $P^{0, r}$ in (3), one may let

$$
p_{i}=\sum_{r \in R_{i}} p_{i}^{r} q_{i}^{r} / \sum_{r \in R_{i}} q_{i}^{r}
$$

By (2) this yields the Lehr index (Lehr, 1885, 39) as a bilateral index in a fixed universe, and a modified GK (MGK) index in a dynamic universe. More generally, replacing (4) by any suitable $p_{i}$ yields a family of GUV indices:

$$
P_{G U V}^{0, t}=\frac{\sum_{i \in U_{t}} p_{i}^{t} q_{i}^{t} / \sum_{i \in U_{t}} p_{i} q_{i}^{t}}{\sum_{i \in U_{0}} p_{i}^{0} q_{i}^{0} / \sum_{i \in U_{0}} p_{i} q_{i}^{0}}=V^{0, t} / Q_{R P}
$$

where $Q_{R P}=\sum_{i \in U_{t}} p_{i} q_{i}^{t} / \sum_{i \in U_{0}} p_{i} q_{i}^{0}$ can be formulated as a reference-price (RP) quantity index. Auer (2014) emphasises the interpretation of $p_{i}$ as an adjustment factor which transforms the transaction quantities $\left(q_{i}^{0}, q_{i}^{t}\right)$ into the "intrinsic-worth units" $\left(\tilde{q}_{i}^{0}, \tilde{q}_{i}^{t}\right)$, where $\tilde{q}_{i}^{r}=p_{i} q_{i}^{r}$ for $r=0, t$. What then matters to the resulting index is only the relevant ratios $p_{i} / p_{j}$ for any $i \neq j$. As pointed out by Dalén (2001), if hedonic regression is used, the factor $p_{i}$ could be determined based on the difference in characteristics between the item and the numeraire (or chosen reference item). In this way the GUV index family can incorporate the hedonic approach.

The test results for the GUV index are similar to the GK index except for the responsiveness. It does not satisfy the identity test $\mathrm{T} 1$ except when $R(0, t)=R_{B}$. It obviously satisfies the fixed-basket test T2. It satisfies the upper bound tests T3 and $\mathrm{t} 3$, provided $p_{i}^{t} \leq p_{i} \leq p_{i}^{0}$ for $i \in U_{R}$. Similarly, it satisfies the lower bound tests T4 and t4 provided $p_{i}^{0} \leq p_{i} \leq p_{i}^{t}$ for $i \in U_{R}$. However, the bilateral GUV index fails the responsiveness test $\mathrm{t} 5$ only in the settings of tests $\mathrm{t} 3$, where $p_{i}=p_{i}^{0}=p_{i}^{t}$ for $i \in U_{0}$ and $p_{i}=p_{i}^{t}$ for $i \in U_{t \backslash 0}$ due to $R=R_{B}$, such that

$$
Q^{0, t}=\frac{\sum_{i \in U_{t}} q_{i}^{t} p_{i}}{\sum_{i \in U_{0}} q_{i}^{0} p_{i}}=\frac{\sum_{i \in U_{t}} q_{i}^{t} p_{i}^{t}}{\sum_{i \in U_{0}} q_{i}^{0} p_{i}^{0}}=V^{0, t} \quad \text { and } \quad P_{G U V}^{0, t}=1,
$$

regardless of $D\left(U_{t \backslash 0}\right)$. Similarly in the setting of test $\mathrm{t} 4$.

\subsection{The WGM Index Family}

A WGM index does not have a direct connection to the expenditure ratio $V^{0, t}$ in general. Like the GUV index, it employs a reference price $p_{j}$, for $j \in U_{R}$, and is given by

$$
P_{W G M}^{0, t}=\frac{\prod_{i \in U_{t}}\left(p_{i}^{t} / p_{i}\right)^{w_{i}^{t}}}{\prod_{i \in U_{0}}\left(p_{i}^{0} / p_{i}\right)^{w_{i}^{0}}}=\left(\frac{\prod_{i \in U_{t}}\left(p_{i}^{t}\right)^{w_{i}^{t}}}{\prod_{i \in U_{0}}\left(p_{i}^{0}\right)^{w_{i}^{0}}}\right) /\left(\frac{\prod_{j \in U_{t}} p_{j}^{w_{j}^{t}}}{\prod_{j \in U_{0}} p_{j}^{w_{j}^{0}}}\right),
$$

with the weights $\sum_{i \in U_{t}} w_{i}^{t}=1$ and $\sum_{i \in U_{0}} w_{i}^{0}=1$. When $R=\{0, t\}$ and $U=U_{0}=U_{t}$, setting $w_{j}^{0}=w_{j}^{t}=\frac{1}{2}\left(q_{j}^{0} p_{j}^{0} / \sum_{i \in U} q_{i}^{0} p_{i}^{0}+q_{j}^{t} p_{j}^{t} / \sum_{i \in U} q_{i}^{t} p_{i}^{t}\right)$ reduces (6) to the Törnqvist 
index. The TPD index (De Haan and Krsinich 2014) is given by

$$
p_{j}=\prod_{r \in R_{j}}\left(\frac{p_{j}^{r}}{P^{0, r}}\right)^{\frac{w_{j}^{r}}{\sum_{b \in R_{j}} w_{j}^{b}}} \text { and } w_{j}^{r}=\frac{q_{j}^{r} p_{j}^{r}}{\sum_{i \in U_{r}} q_{i}^{r} p_{i}^{r}} .
$$

Fattore (2010) considers the axiomatic properties of the geo-logarithmic family (GLF) of indices. The GLF index is a special case of the WGM index, with fixed universe $U_{0}=U_{t}$ and the same weights at both periods 0 and $t$, i.e., $w_{i}=w_{i}^{0}=w_{i}^{t}$, where $w_{i}$ can depend on data at both 0 and $t$.

The WGM index (6) does not satisfy the identity test T1 except when $R(0, t)=R_{B}$, since otherwise one can not ensure $p_{j}=p_{j}^{0}=p_{j}^{t}$ in a dynamic universe. It generally does not satisfy the fixed-basket test $\mathrm{T} 2$ due to the lack of direct connection to $V^{0, t}$. It satisfies the upper bound test $\mathrm{T} 3$, provided $p_{i}^{t} \leq p_{i} \leq p_{i}^{0}$, such that

$$
P_{W G M}^{0, t}=\left(\prod_{i \in U_{t}}\left(p_{i}^{t} / p_{i}\right)^{w_{i}^{t}}\right)\left(\prod_{i \in U_{0}}\left(p_{i} / p_{i}^{0}\right)^{w_{i}^{0}}\right) \leq 1 .
$$

Similarly, it satisfies the lower bound test T3, provided $p_{i}^{0} \leq p_{i} \leq p_{i}^{t}$. Under the settings of tests $\mathrm{t} 3$ and $\mathrm{t} 4$, we have $P_{W G M}^{0, t}=P_{W G M}^{0, t}\left(D_{0 t}\right)=1$, provided $p_{j}=p_{j}^{0}=p_{j}^{t}$, such that it satisfies these tests while failing the responsiveness test t5 at the same time.

\subsection{The GEKS Index}

Provided $R(0, t)=R_{M}$ and $t \geq 2$, the GEKS index from 0 to $r$, for $0<r \leq t$, is given by

$$
P_{G E K S}^{0, r}=\left(\prod_{s=0}^{t} P^{0, s} P^{s, r}\right)^{\frac{1}{t+1}}=\left(\left(P^{0, r}\right)^{2} \prod_{s \neq 0, r} P^{0, s} P^{s, r}\right)^{\frac{1}{t+1}}
$$

For any $r<t$, it involves indirect comparisons via the periods outside $\{0, \ldots, r\}$. For example, if $t=2$ and $r=1$, we have $P^{0,1}=\left(\left(P^{0,1}\right)^{2} P^{0,2} P^{2,1}\right)^{\frac{1}{3}}$ where both $P^{0,2}$ and $P^{2,1}$ are only available in period 2 but not period 1 . Therefore, in practice, the disseminated GEKS index, denoted by $\hat{P}_{G E K S}^{0, r}$ is always the one with $r=t$ in (7). It is intransitive since, for any $0<r<t$,

$$
\begin{aligned}
\hat{P}_{G E K S}^{0, t} & =\left(\left(P^{0, t}\right)^{2} \prod_{0<s<t} P^{0, s} P^{s, t}\right)^{\frac{1}{t+1}}=\left(\left(P^{0, t}\right)^{2} P^{0, r} P^{r, t} \prod_{0<s<t ; s \neq r} P^{0, s} P^{s, t}\right)^{\frac{1}{t+1}} \\
& \neq \hat{P}_{G E K S}^{0, r} \hat{P}_{G E K S}^{r, t}=\left(\left(P^{0, r}\right)^{2} \prod_{0<s<r} P^{0, s} P^{s, r}\right)^{\frac{1}{r+1}}\left(\left(P^{r, t}\right)^{2} \prod_{r<s<t} P^{r, s} P^{s, t}\right)^{\frac{1}{t-r+1}},
\end{aligned}
$$

where the set of reference periods is $R(0, t)=\{0,1, \ldots, t\}$ for $\hat{P}_{G E K S}^{0, t}$, it is $R(0, r)=$ $\{0,1, \ldots, r\}$ for $\hat{P}_{G E K S}^{0, r}$ and $R(r, t)=\{r, r+1, \ldots, t\}$ for $\hat{P}_{G E K S}^{r, t}$, according to the default 
choice of $R_{M}$. For example, for $t=2$, the three GEKS indices are intransitive, where

$$
\begin{aligned}
& \hat{P}_{G E K S}^{0,2}=\left(\left(P^{0,2}\right)^{2} P^{0,1} P^{1,2}\right)^{\frac{1}{3}}, \quad \hat{P}_{G E K S}^{0,1}=\left(\left(P^{0,1}\right)^{2}\right)^{\frac{1}{2}}=P^{0,1} \\
& \hat{P}_{G E K S}^{1,2}=\left(\left(P^{1,2}\right)^{2}\right)^{\frac{1}{2}}=P^{1,2}
\end{aligned}
$$

It is, of course, possible to use a different $R_{M}$, such as a 13-month window that moves with $t$. But the GEKS remains intransitive, because $P^{0, r}$ calculated with $R_{M}=\{r-12$, $r-11, \ldots, r\}$ is different from $P^{0, r}$ calculated with $R_{M}=\{t-12, t-11, \ldots, t\}$, provided $t-12 \leq 0<r<t$.

The components in (7) can be any bilateral time-reversible index. Now that the reference universe of the GEKS index by definition cannot be $U_{R_{B}}$ for $t>1$, it generally does not pass any other tests than the responsiveness test $\mathrm{T} 5$.

\section{A Reference-Quantity-Price Index Family}

None of the indices considered in Section 3 satisfies all the five tests proposed in this article. Two observations seem worth noting. First, a multilateral index generally does not satisfy the identity test T1 nor the fixed-basket test T2. However, we do not therefore conclude that a bilateral index is preferable to a multilateral index in practice, since none of them are perfect and it is possible to compensate for a small shortcoming in one respect with better properties in others. Second, there is a tension between the bound tests $\mathrm{t} 3$ and $\mathrm{t} 4$ on the one hand, and the responsiveness test $\mathrm{t} 5$ on the other hand. As a potential means to a resolution, we outline below a large index family, which includes the GUV index family as a subclass. Let

$$
P_{R Q P}^{0, t}=\left(P_{R Q}^{0, t}\right)^{1-\alpha}\left(P_{G U V}^{0, t}\right)^{\alpha}
$$

where $\alpha$ is a constant of choice, for $0 \leq \alpha \leq 1$, and $P_{G U V}^{0, t}$ is given by (5), and the reference-quantity index $P_{R Q}^{0, t}$ is given by

$$
P_{R Q}^{0, t}=\sum_{i \in U_{0 \cup t}} q_{i} p_{i}^{t} / \sum_{i \in U_{0 \cup t}} q_{i} p_{i}^{0}
$$

where $U_{0 \cup t}=U_{0} \cup U_{t}$, and $q_{i}$ is a reference-quantity for $i \in U_{0 \cup t}$. We shall refer to (8) as the reference-quantity-price $(R Q P)$ index. It reduces to a GUV index if $\alpha=1$.

Provided $0<\alpha<1$, an RQP index makes use of both reference quantities $q_{i}$ and reference prices $p_{i}$. The expression (8) shows it as a weighted geometric mean of two price indices. It can equally be expressed as deflating the expenditure ratio $V^{0, t}$ by a weighted geometric mean of two quantity indices $Q_{R P}^{0, t}$ and $V^{0, t} / P_{R Q}^{0, t}$, i.e.,

$$
P_{R Q P}^{0, t}=V^{0, t} /\left[\left(Q_{R P}^{0, t}\right)^{\alpha}\left(V^{0, t} / P_{R Q}^{0, t}\right)^{1-\alpha}\right]
$$


In particular, at $\alpha=0.5$, the RQP index can be considered to generalise the Fisher index, defined in the special case of $U=U_{0}=U_{t}=U_{0 \cup t}$ and $R(0, t)=R_{B}$. That is,

$$
P_{L}^{0, t} P_{P}^{0, t}=P_{L}^{0, t} V^{0, t} / Q_{L}^{0, t}=P_{P}^{0, t} V^{0, t} / Q_{P}^{0, t}
$$

where $P_{L}^{0, t}$ is the Laspeyres price index given as $P_{R Q}^{0, t}$ with $q_{i}=q_{i}^{0}$, and $P_{L}^{0, t}$ is the Paasche price index given as $P_{R Q}^{0, t}$ with $q_{i}=q_{i}^{t}$, and $Q_{L}^{0, t}$ is the Laspeyres quantity index given as $Q_{R P}^{0, t}$ with $p_{i}=p_{i}^{0}$, and $Q_{P}^{0, t}$ is the Paasche quantity index given as $Q_{R P}^{0, t}$ with $p_{i}=p_{i}^{t}$.

There are many possible choices for the reference price in $P_{G U V}^{0, t}$ and the reference quantity in $P_{R Q}^{0, t}$. In the existing though limited studies and practices of $P_{G U V}^{0, t}$, the reference price $p_{i}$ is usually set to be the unit-value price of item $i$ over the chosen reference universe, that is calculated over the periods in which the item is available. However, in certain situations, one may instead consider using the introductory price or another representative price. When it comes to the reference quantity $q_{i}$ in $P_{R Q}^{0, t}$, one can obviously extend the various arithmetic and geometric means defined for the fixed universe. Or, one may set $q_{i}$ to be the ratio between the average expenditure of item $i$ and the reference price $p_{i}$ calculated for the GUV-counterpart. In particular, we believe it will be necessary to study these questions together with the formation of homogeneous products, which are defined at a level that is between the items identified by (GTIN, outlet) and the elementary aggregate. However, it is beyond the scope of this paper to address these issues.

The RQ index $P_{R Q}^{0, t}$ satisfies obviously the identity test T1. It satisfies the fixed-basket test T2 provided $R(0, t)=R_{B}$. It satisfies the responsiveness test T5, as long as $q_{i}>0$ for $i \in U_{0 \cup t} \backslash U_{0 t}$. Moreover, it provides a means to resolve the tension between the bound tests $\mathrm{t} 3$ and $\mathrm{t} 4$ and the responsiveness test $\mathrm{t} 5$. To satisfy the test $\mathrm{t} 5$ in the setting of the test t3, where $U_{0 \cup t}=U_{0} \cup U_{t \backslash 0}, p_{i}^{0}=p_{i}^{t}$ for $i \in U_{0}$ and $q_{i}^{t}>0$ for $i \in U_{t \backslash 0}$, we require $P_{R Q}^{0, t}<1$. Since $\sum_{U_{0}} q_{i} p_{i}^{t}=\sum_{U_{0}} q_{i} p_{i}^{0}$ regardless of the choice of $q_{i}$ for $i \in U_{0}$, we need $\sum_{i \in U_{t \backslash 0}} q_{i} p_{i}^{0}>\sum_{i \in U_{t \backslash 0}} q_{i} p_{i}^{t}$, given any choice of $q_{i}$ for $i \in U_{t \backslash 0}$. This can be achieved by imputing a price $\hat{p}_{i}^{0}$, where $\hat{p}_{i}^{0}>p_{i}^{t}$ for $i \in U_{t \backslash 0}$. Provided such $\hat{p}_{i}^{0}$, the imputed referencequantity expenditure in period 0 would be higher than the reference-quantity expenditure in period $t$, that is

$$
\sum_{U_{0}} q_{i} p_{i}^{0}+\sum_{U_{t \backslash 0}} q_{i} \hat{p}_{i}^{0}>\sum_{U_{0}} q_{i} p_{i}^{0}+\sum_{U_{t \backslash 0}} q_{i} p_{i}^{t}=\sum_{U_{0}} q_{i} p_{i}^{t}+\sum_{U_{t \backslash 0}} q_{i} p_{i}^{t}
$$

It follows that the imputed $P_{R Q}^{0, t}$ is less than 1 , which satisfies the upper bound test $t 3$ and the responsiveness test t5 at the same time. Similarly, the imputed RQ index $P_{R Q}^{0, t}$ satisfies jointly the tests $\mathrm{t} 4$ and $\mathrm{t} 5$, provided $\hat{p}_{i}^{t}>p_{i}^{0}$ for $i \in U_{0 \backslash t}$.

Imputation seems a natural remedy for the RQ index because, unlike the MGK, GUV or WGM index, it lacks otherwise a mechanism that accounts for the differing sizes of the item universes $U_{0}$ and $U_{t}$. The inclusion of $\sum_{U_{t \backslash 0}} q_{i} \hat{p}_{i}^{0}$ or $\sum_{U_{0 \backslash t}} q_{i} \hat{p}_{i}^{t}$ can be considered as a means to incorporate a dynamic basket from the COGI perspective, or to align the utility over time from the COLI perspective. In the setting of test T3, where $p_{i}^{t}<p_{i}^{0}$ for at least some $i \in U_{0}$, we have $\sum_{U_{0}} q_{i} p_{i}^{t}<\sum_{U_{0}} q_{i} p_{i}^{0}$ regardless of the choice of $q_{i}$ for $i \in U_{0}$. It follows that the imputed $P_{R Q}^{0, t}$ satisfies the test T3, provided any $\hat{p}_{i}^{0} \geq p_{i}^{t}$ for $i \in U_{t \backslash 0}$, 
including the choice of $\hat{p}_{i}^{0}=p_{i}^{t}$. Similarly, it satisfies the lower bound test T4, provided $\hat{p}_{i}^{t} \geq p_{i}^{0}$ for $i \in U_{0 \backslash t}$, including the choice of $\hat{p}_{i}^{t}=p_{i}^{0}$.

The test results of the RQP index can be deduced from those of $P_{G U V}^{0, t}$ and $P_{R Q}^{0, t}$. Thus, given a judicious choice of the imputed $P_{R Q}^{0, t}$, it can potentially satisfy all the five tests.

\section{Concluding Remarks}

The proposed set of tests provide a rigorous diagnostic for whether an index can be considered completely appropriate in a dynamic item universe, as well as pointing towards the directions of possible remedies. The RQP index family can potentially satisfy all the tests. It extends the GUV index family that has received much attention in the recent years. But more research is needed regarding the imputation method and the mixing weight $\alpha$.

We reiterate that failing one or more tests does not in itself make an index unacceptable in practice, because not exactly satisfying a test does not mean that it is not satisfied approximately, and it is possible for an index to compensate for a small shortcoming in one respect with better properties in others. Moreover, the test approach does not directly provide the solutions to the many other choices one necessarily has to make in practice. These include the use of fixed base period versus moving base and indirect measurement of the short-term price index, the aggregation structure of the CPI including the formation of homogeneous products, the balance between automatic item-matching and manual intervention, the decision between bilateral and multilateral indices in a given CPI subuniverse, and so on. For these reasons, we believe it is important, in future research, to develop sensible empirical criteria, regarding when an index based on the unit value price data can be considered acceptable for practical purposes.

\section{References}

ABS. 2016. Making Greater Use of Transactions Data to Compile the Consumer Price Index, Australia. The Australian Bureau of Statistics (Catalogue No. 6401.0.60.003). Available at: https://www.abs.gov.au/ausstats/abs@.nsf/mf/6401.0.60.003 (accessed June 2019).

Auer, von L. 2014. "The Generalized Unit Value Index Family." Review of Income and Wealth 60: 843-861. Doi: https://doi.org/10.1111/roiw.12042.

Balk, B.M. 1996. "A Comparison of Ten Methods for Multilateral International Price and Volume Comparison.” Journal of Official Statistics 12: 199-222. Available at: https://search.proquest.com/docview/1266834049?pq-origsite=gscholar (accessed June 2019).

Balk, B.M. 2001. Aggregation Methods in International Comparisons: What Have We Learned? ERIM Report, Erasmus Research Institute of Management, Erasmus University Rotterdam. Available at: https://papers.ssrn.com/sol3/papers.cfm?abstract $\mathrm{id}=370897$ (accessed June 2019).

Chessa, A.G. 2016. "A New Methodology for Processing Scanner Data in the Dutch CPI." Eurostat review of National Accounts and Macroeconomic Indicators 1: 49-69. Available at: https://ec.europa.eu/eurostat/cros/system/files/euronaissue1-2016-art2.pdf (accessed June 2019). 
Chessa, A.G., J. Verburg, and L. Willenborg. 2017. A Comparison of Price Index Methods for Scanner Data. Paper presented at the fifteenth Ottawa Group meeting, 10-12 May 2017, Eltville, Germany. Available at: http://www.ottawagroup.org/ Ottawa/ottawagroup.nsf/4a256353001af3ed4b2562bb00121564/1ab31c25da944ff 5ca25822c00757f87/\$FILE/A\%20comparison\%20of\%20price\%20index\%20methods $\% 20$ for $\% 20$ scanner $\% 20$ data\%20-Antonio\%20Chessa, \%20Johan \%20Verburg, $\% 20$ Leon\%20Willenborg\%20-Paper.pdf (accessed June 2019).

CPI Manual. 2004. Consumer Price Index Manual: Theory and Practice. International Labour Organization. Available at: http://www.ilo.org/public/english/bureau/stat/ guides/cpi/index.htm (accessed June 2019).

Dalén, J. 2001. Statistical Targets for Price Indexes in Dynamic Universes. Paper presented at the sixth Ottawa Group meeting, 2-6 April 2001, Canberra, Australia. Available at: http://www.ottawagroup.org/Ottawa/ottawagroup.nsf/home/Meeting + 6/\$file/2001 \%206th\%20Meeting\%20-\%20Dal\%C3\%A9n\%20J\%C3\%B6rgen\%20-\%20Statistical $\% 20$ targets\%20for\%20price\%20indexes\%20in\%20dynamic\%20universes.pdf.

Dalén, J. 2017. Unit Values in Scanner Data Some Operational Issues. Paper presented at the fifteenth Ottawa Group meeting, 10-12 May 2017, Eltville, Germany. Available at: http://www.ottawagroup.org/Ottawa/ottawagroup.nsf/4a256353001af3ed4b2562bb 00121564/1ab31c25da944ff5ca25822c00757f87/\$FILE/Unit\%20values\%20in\%20 scanner $\% 20$ data $\% 20 \%$ E2\% $80 \% 93 \% 20$ some $\% 20$ operational $\% 20$ issues $\% 20-\% 20 \mathrm{~J} \%$ C3\%B6rgen\%20Dal\%C3\%A9n\%20-Paper.pdf (accessed June 2019).

De Haan, J. and F. Krsinich. 2014. "Scanner Data and the Treatment of Quality Change in Nonrevisable Price Indexes.” Journal of Business \& Economic Statistics 32: 341-358. Doi: https://doi.org/10.1080/07350015.2014.880059.

De Haan, J. 2001. Generalized Fisher Price Indexes and the Use of Scanner Data in the CPI. Paper presented at the sixth Ottawa Group meeting, 2-6 April 2001, Canberra, Australia. Available at: http://www.ottawagroup.org/Ottawa/ottawagroup.nsf/home/ Meeting + 6/\$file/2001\%206th\%20Meeting\%20-\%20de $\% 20$ Haan $\% 20$ Jan $\% 20-\% 20$ Generalised $\% 20$ Fisher $\% 20$ Price $\% 20$ Indexes $\% 20$ and $\% 20$ the $\% 20$ Use $\% 20$ of $\% 20$ Scanner\%20Data\%20in\%20the\%20CPI.pdf (accessed June 2019).

Diewert, E.W. 1999. "Axiomatic and Economic Approaches to International Comparisons." In International and Interarea Comparisons of Income, Output, and Prices, edited by A. Heston and R.E. Lipsey, 13-87. Studies in Income and Wealth, Vol. 61. Chicago: University of Chicago Press.

Diewert, E.W. and K.J. Fox. 2017. Substitution Bias in Multilateral Methods for CPI Construction Using Scanner Data. Paper presented at the fifteenth Ottawa Group meeting, 10-12 May 2017, Eltville, Germany. Available at: http://irs.princeton.edu/ sites/irs/files/Diewert\%20and\%20Fox\%20Substitution\%20Bias\%20and\%20MultilateralMethodsForCPI_DP17-02_March23.pdf (accessed June 2019).

Fattore, M. 2010. “Axiomatic Properties of Geo-logarithmic Price Indices.” Journal of Econometrics 156: 344-353. Doi: https://doi.org/10.1016/j.jeconom.2009.11.004.

Fisher, I. 1922. The Making of Index Numbers. Boston: Houghton-Mifflin.

Geary, R.C. 1958. "A Note on Comparisons of Exchange Rates and Purchasing Power Between Countries.” Journal of the Royal Statistical Society, Series A 121: 97-99. Available at: https://www.jstor.org/stable/pdf/2342991.pdf (accessed June 2019). 
Ivancic, L., K.J. Fox, and E.W. Diewert. 2011. "Scanner Data, Time Aggregation and the Construction of Price Indexes." Journal of Econometrics 161: 24-35. Doi: https://doi.org/10.1016/j.jeconom.2010.09.003.

Lehr, J. 1885. Beitrüge zur Statistik der Preise insbesondere des Geldes und des Holzes, F.D. Sauerländer Verlag, Frankfurt a. M.

Schultze, C.L. and C. Mackie. 2002. At What Price?: Conceptualizing and Measuring Cost-of-Living and Price Indexes. The National Academies Press. Doi: http://dx.doi. org/10.17226/10131.

Zhang, L.C., I. Johansen, and R. Nygaard. 2017. Testing Unit Value Data Price Indices.

Paper presented at the fifteenth Ottawa Group meeting, 10-12 May 2017, Eltville, Germany. Available at: http://www.ottawagroup.org/Ottawa/ottawagroup.nsf/ 4a256353001af3ed4b2562bb00121564/1ab31c25da944ff5ca25822c00757f87/\$FILE/ Testing\%20unit\%20value\%20data\%20price\%20indices\%20-\%20Li-Chun\%20Zhang, \%20Ingvild\%20Johansen,\%20Ragnhild\%20Nygaard\%20-\%20Paper.pdf (accessed June 2019).

Received November 2018

Revised March 2019

Accepted May 2019 